\title{
Optimal Quantization in Energy-Constrained Sensor Networks under Imperfect Transmission
}

\author{
Li Zhang ${ }^{1,2}$, Tao Cui ${ }^{1}$, Tracey $\mathrm{Ho}^{1}$ \\ ${ }^{1}$ Department of Electrical Engineering \\ California Institute of Technology \\ Pasadena CA 91125, USA \\ Email: \{lizhang,taocui,tho\}@ caltech.edu
}

\author{
Xian-Da Zhang ${ }^{2}$ \\ ${ }^{2}$ Department of Automation, Tsinghua University \\ Beijing 100084, China \\ Email: zxd-dau@mail.thu.edu.cn
}

\begin{abstract}
This paper addresses the optimization of quantization at local sensors under strict energy constraint and imperfect transmission to improve the reconstruction performance at the fusion center in the wireless sensor networks (WSNs). We present optimized quantization scheme including the optimal quantization bit rate and the optimal transmission power allocation among quantization bits for BPSK signal and binary orthogonal signal with envelope detection, respectively. The optimization of the quantization is formulated as a convex problem and the optimal solution is derived analytically in both cases. Simulation results demonstrate the effectiveness of our proposed quantization schemes.
\end{abstract}

\section{INTRODUCTION}

Parameter estimation by a set of distributed sensors and a fusion center $(\mathrm{FC})$ is frequently encountered in the wireless sensor network (WSN) applications [1]. Constrained by limited power and bandwidth resources, sensors should perform local quantization to their observations before transmission [2]. At the FC, sensor observations are reconstructed and combined to produce a final estimate of the unknown parameter.

Some previous works [3]-[5] assume error-free transmission between sensors and the FC. [6]-[10] model the wireless links between sensors and the FC as additive white Gaussian noise (AWGN) channels. [6] derives an estimate scheme based on 1-bit quantized observation. The optimal power scheduling problems among sensors are investigated in [7] [8] with different assumptions about the sensor observation noise. Training sequence is used in [9] to estimate the unknown channel.

Different from these works, this paper addresses the optimization of the quantization scheme per sensor including the quantization bit rate and the transmission energy allocation among quantization bits under strict energy constraint and imperfect transmission. A quantization scheme is proposed in [10], which minimizes the upper bound of the reconstruction mean-absolute error (MBE), which is called the MBE quantization scheme below.

In contrast, we propose a quantization scheme which minimizes an upper bound of the reconstruction MSE for BPSK signal and binary orthogonal signal with envelope detection, respectively. The optimal transmission power allocation among

This work was supported in part by the Major Program of the National Natural Science Foundation of China under Grant 60675002 and Caltech's Lee Center for Advanced Networking. quantization bits is formulated as a convex problem and the optimal solution is derived analytically in both cases. Simulation results show that the proposed quantization schemes achieve significant reduction in reconstruction MSE compared with the MBE quantization scheme and the quantization scheme with uniform energy allocation for either BPSK signal or binary orthogonal signal with envelope detection .

\section{Problem Formulation}

We consider that each sensor in the network makes a local observation $y$ and wishes to transmit it to FC. Suppose that the observation $y$ is bounded to $[-T, T]$ with variance $\sigma^{2}$, where $T$ is known and decided by the sensor's dynamic range. We can express $y$ as

$$
y=2 T \sum_{n=1}^{\infty} b_{n} 2^{-n}-T .
$$

Here, we consider the $N$-bit uniform quantization, which is widely used in energy-constrained sensor networks. Denote $\bar{y}$ as the $N$-bit quantized version of $y$, where

$$
\bar{y}=2 T \sum_{n=1}^{N} b_{n} 2^{-n}-T .
$$

Due to the constrained power, only the quantization bits $\left\{b_{n}\right\}_{n=1}^{N}$ will be transmitted to the FC. We model the wireless link between the sensor and the FC as an AWGN channel. Here, the uncoded transmission strategy is used. Denote the total transmission energy as $\xi$ and the channel noise variance as $\sigma_{n}^{2}$. At the FC, the total receiving signal-to-noise ratio (SNR) is defined by $\gamma:=\xi / \sigma_{n}^{2}$. We assume the transmission energy for $b_{n}$ is a fraction $x_{n}$ of the total energy $\xi$ for $n=1, \ldots, N$, and the quantization bits experience independent channels. Denote $b_{n}^{\prime}$ as the demodulated version of $b_{n}$ at the FC. Then the SNR of $b_{n}^{\prime}$ is $x_{n} \gamma$. Denote $p_{n}$ as the bit error rate of $b_{n}$, that is $p_{n}:=P\left(b_{n}^{\prime} \neq b_{n}\right)$.

At the FC, $y$ can be reconstructed as

$$
\bar{y}^{\prime}=2 T \sum_{n=1}^{N} b_{n}^{\prime} 2^{-n}-T .
$$

Our goal in this paper is to optimize the quantization scheme under the total energy constraint $\xi$ in order to minimize 
the reconstruction MSE at the FC. The quantization scheme includes the quantization bit rate $N$ and the transmission energy allocation among bits $\boldsymbol{x}=\left[x_{1}, \ldots, x_{N}\right]$.

\section{Optimal Quantization Schemes}

Firstly, we obtain an upper bound of the reconstruction MSE. At the FC, the reconstruction error can be expressed as

$$
y-\bar{y}^{\prime}=(y-\bar{y})+\left(\bar{y}-\bar{y}^{\prime}\right)
$$

where the first part is the quantization error and the second part is the demodulation error. Using (4) and the Cauchy-Schwartz inequality, we can bound the reconstruction MSE as

$$
E\left|y-\bar{y}^{\prime}\right|^{2} \leq 2 E|y-\bar{y}|^{2}+2 E\left|\bar{y}-\bar{y}^{\prime}\right|^{2} .
$$

According to (2) and (3), we obtain

$$
\begin{aligned}
& E\left|\bar{y}-\bar{y}^{\prime}\right|^{2}=4 T^{2} E\left|\sum_{n=1}^{N}\left(b_{n}-b_{n}^{\prime}\right) 2^{-n}\right|^{2} \\
& \leq 4 T^{2} E\left(\sum_{n=1}^{N}\left|b_{n}-b_{n}^{\prime}\right| 2^{-n}\right)^{2} \\
& =4 T^{2}\left(\sum_{n=1}^{N} E\left|b_{n}-b_{n}^{\prime}\right|^{2} 2^{-2 n}\right. \\
& \left.+\sum_{n \neq m}^{N} E\left|b_{n}-b_{n}^{\prime}\right| E\left|b_{m}-b_{m}^{\prime}\right| 2^{-n-m}\right),
\end{aligned}
$$

where the last step is due to the assumption that the quantization bits experience independent channels.

Recalling that $\left|b_{n}-b_{n}^{\prime}\right|$ is a $\{0,1\}$ Bernoulli random variable, we have $E\left|b_{n}-b_{n}^{\prime}\right|^{2}=E\left|b_{n}-b_{n}^{\prime}\right|=p_{n}$. Thus (8) can be expressed as

$$
E\left|\bar{y}-\bar{y}^{\prime}\right|^{2} \leq 4 T^{2}\left(\sum_{n=1}^{N} p_{n} 2^{-2 n}+\sum_{n \neq m}^{N} p_{n} p_{m} 2^{-n-m}\right) .
$$

From (6) we find that the demodulation MSE is more dependent on the demodulation accuracy of the leading bits of $y$, and less dependent on the accuracy of its trailing bits. Intuitively, it is reasonable to assume that the optimal quantization scheme allocates more transmission energy to the leading bits of $y$, and less energy for its trailing bits, that is $x_{m} \geq x_{n}$ if $m \leq n$. Then we have

$$
p_{m} \leq p_{n}, \quad \text { if } \quad m \leq n,
$$

which can be easily shown by contradiction.

Using (10) and the fact $p_{m} \leq 1$, we can further bound the second part of (9) as

$$
\begin{gathered}
\sum_{n \neq m}^{N} p_{n} p_{m} 2^{-n-m} \leq \sum_{n=1}^{N} p_{n} 2^{-n}\left(p_{n} \sum_{m=1}^{n-1} 2^{-m}+\sum_{m=n+1}^{N} 2^{-m}\right) \\
=\sum_{n=1}^{N}\left[p_{n} 2^{-n}\left(2^{-n}-2^{-N}\right)+p_{n}^{2} 2^{-n}\left(1-2^{1-n}\right)\right] .
\end{gathered}
$$

Substituting (11) into (9), we have

$$
\begin{gathered}
E\left|\bar{y}-\bar{y}^{\prime}\right|^{2} \leq 4 T^{2} \sum_{n=1}^{N}\left[p_{n} 2^{1-2 n}\left(1-2^{n-N-1}\right)\right. \\
\left.+p_{n}^{2} 2^{-n}\left(1-2^{1-n}\right)\right]
\end{gathered}
$$

It has been shown in [5] that the quantization error satisfies

$$
E|y-\bar{y}|^{2} \leq \sigma^{2}+\frac{T^{2}}{3} 2^{-2 N}
$$

According to (12) and (13), we can finally bound the reconstruction MSE as [c.f. (5)]

$$
\begin{aligned}
E\left|y-\bar{y}^{\prime}\right|^{2} & \leq 2 \sigma^{2}+\frac{T^{2}}{3} 2^{1-2 N}+8 T^{2} \sum_{n=1}^{N}\left[p_{n} 2^{1-2 n}\right. \\
& \left.\cdot\left(1-2^{n-N-1}\right)+p_{n}^{2} 2^{-n}\left(1-2^{1-n}\right)\right] \\
& :=f(N ; \mathbf{x})
\end{aligned}
$$

The power scheduling among quantization bits is constructed as follows:

$$
\begin{array}{ll}
\min & f(N ; \boldsymbol{x}), \\
\text { s.t. } & x_{n} \geq 0, \quad n=1, \ldots, N \\
& \sum_{n=1}^{N} x_{n}=1
\end{array}
$$

The optimal solution of (15) and the minimum of the objective function $f(N ; \boldsymbol{x})$ are functions of the quantization bit rate $N$. We denote the optimal solution of $\boldsymbol{x}$ as $\boldsymbol{x}_{N}^{*}=$ $\left[x_{N, 1}^{*}, x_{N, 2}^{*}, \ldots, x_{N, N}^{*}\right]$ and the minimum of the objective function as $f\left(N ; \boldsymbol{x}_{N}^{*}\right)$. The optimal quantization bit rate which minimizes the upper bound of the reconstruction MSE can then be obtained by

$$
N_{o p t}=\arg \min _{N} f\left(N ; \boldsymbol{x}_{N}^{*}\right) .
$$

When $N$ is fixed, to solve the convex problem (15), we can write the Lagrangian function $G$ as

$G\left(\boldsymbol{x}_{N}^{*}, \lambda, \boldsymbol{\mu}\right)=f\left(N ; \boldsymbol{x}_{N}^{*}\right)+\lambda\left(\sum_{n=1}^{N} x_{N, n}^{*}-1\right)-\sum_{n=1}^{N} \mu_{n} x_{N, n}^{*}$. 


\section{A. BPSK over AWGN Channels}

The bit error rate $p_{n}$ for BPSK signal over AWGN channels is given by [11] as

$$
p_{n}=Q\left(\sqrt{x_{N, n}^{*} \gamma}\right)
$$

where $Q(\cdot)$ is Gaussian tail function.

We then have

$$
\begin{aligned}
f\left(N ; \boldsymbol{x}_{N}^{*}\right)= & 2 \sigma^{2}+\frac{T^{2}}{3} 2^{1-2 N}+8 T^{2} \sum_{n=1}^{N}\left[Q\left(\sqrt{x_{N, n}^{*} \gamma}\right) 2^{1-2 n}\right. \\
& \left.\cdot\left(1-2^{n-N-1}\right)+Q\left(\sqrt{x_{N, n}^{*} \gamma}\right)^{2} 2^{-n}\left(1-2^{1-n}\right)\right] .
\end{aligned}
$$

In this case, the second derivative of $f\left(N ; \boldsymbol{x}_{N}^{*}\right)$ with respect to $x_{N, n}^{*}$ is

$$
\begin{aligned}
\frac{\partial^{2} f\left(N ; \boldsymbol{x}_{N}^{*}\right)}{\partial x_{N, n}^{*}{ }^{2}} & =T^{2} \sqrt{\frac{\gamma}{2 \pi x_{N, n}^{*}}} 2^{4-2 n} e^{-\frac{x_{N, n}^{*} \gamma}{2}}\left\{\frac{1}{2}\left(\frac{1}{x_{N, n}^{*}}+\gamma\right)\right. \\
& \cdot\left[1-2^{n-N-1}+Q\left(\sqrt{x_{n} \gamma}\right)\left(2^{n}-2\right)\right] \\
& \left.+\sqrt{\frac{\gamma}{2 \pi x_{N, n}^{*}}}\left(2^{n}-2\right) e^{-\frac{x_{N, n}^{*} \gamma}{2}}\right\} \\
& >0 .
\end{aligned}
$$

Thus the optimal problem (15) is convex with respect to $x_{N, n}^{*}$ for BPSK signal over AWGN channels.

The associated set of KKT conditions then read [12]

$$
\begin{gathered}
T^{2} \sqrt{\frac{\gamma}{2 \pi x_{N, n}^{*}} e^{-\frac{1}{2} x_{N, n}^{*} \gamma} 2^{4-2 n}\left[1-2^{n-N-1}+\right.} \\
\left.Q\left(\sqrt{x_{N, n}^{*} \gamma}\right)\left(2^{n}-2\right)\right]-\lambda+\mu_{n}=0 \\
\lambda\left(\sum_{n=1}^{N} x_{N, n}^{*}-1\right)=0 \\
\sum_{n=1}^{N} x_{N, n}^{*}=1 \\
\mu_{n} x_{N, n}^{*}=0, \quad n=1, \ldots, N \\
\lambda \geq 0 \quad \mu_{n} \geq 0 \quad x_{N, n}^{*} \geq 0 \quad n=1, \ldots, N
\end{gathered}
$$

We notice that if $\lambda=0$, from (19) it follows that $\mu_{n}<0$ for all $n$. Then using (22), we have $x_{N, n}^{*}=0$ for all $n$, which violates the condition (21). Thus, it must holds that $\lambda>0$.

Now we proceed to solve the KKT systems. Define function $\psi\left(x_{N, n}^{*}\right)$ as

$$
\begin{aligned}
\psi\left(x_{N, n}^{*}\right):=T^{2} \sqrt{\frac{\gamma}{2 \pi x_{N, n}^{*}}} e^{-\frac{1}{2} x_{N, n}^{*} \gamma}\left[1-2^{n-N-1}\right. \\
\\
\left.+Q\left(\sqrt{x_{N, n}^{*} \gamma}\right)\left(2^{n}-2\right)\right] .
\end{aligned}
$$

It is easy to show $\partial \psi\left(x_{N, n}^{*}\right) / \partial x_{N, n}^{*}<0$, which implies that $\psi\left(x_{N, n}^{*}\right)$ is strictly decreasing with respect to $x_{N, n}^{*}$. Due to (19), we can express the optimal solution as

$$
x_{N, n}^{*}=\psi^{-1}\left(2^{2 n-4}\left(\lambda-\mu_{n}\right)\right) .
$$

Since the domain of $\psi\left(x_{N, n}^{*}\right)$ defined in (24) is $(0,+\infty)$, we must have $\mu_{n}=0$ for all $n$ to satisfy the complementary slackness conditions in (22). Therefore, we obtain the final solution of (15) as

$$
x_{N, n}^{*}=\psi^{-1}\left(2^{2 n-1} \lambda\right),
$$

where $\lambda$ is a constant chosen to enforce the constraint $\sum_{n=1}^{N} x_{N, n}^{*}=1$.

Since $\psi\left(x_{N, n}^{*}\right)$ is monotonically decreasing with respect to $x_{N, n}^{*}, \sum_{n=1}^{N} x_{N, n}^{*}$ is a monotonically decreasing function with respect to $\lambda$. Thus, we can use some efficient search algorithms to find the proper $\lambda$ which makes the constraint satisfied, such as the bisearch algorithm.

The optimal energy allocation in (26) intuitively shows that more energy should be allocated to the leading bits, while less should be allocated to the trailing ones, which indicates our original assumption in (10) is reasonable.

Finally, the optimal solution $\left\{N_{o p t}, \boldsymbol{x}_{N_{o p t}}^{*}\right\}$ can be obtained based on (16).

\section{B. Binary Orthogonal Signal with Envelope Detection}

Consider the binary orthogonal signals such as binary frequency-shift keying (FSK) or pulse-position modulation (PPM), which can be demodulated using non-coherent envelope detection. The bit error rate is [9, pp. 307-310]

Then we have

$$
p_{n}=\frac{1}{2} e^{-\frac{x_{N, n}^{*} \gamma}{4}}
$$

$$
\begin{aligned}
f\left(N ; \boldsymbol{x}_{N}^{*}\right)= & 2 \sigma^{2}+\frac{T^{2}}{3} 2^{1-2 N}+8 T^{2} \sum_{n=1}^{N}\left[e^{-\frac{x_{N, n}^{*} \gamma}{4}} 2^{-2 n}\right. \\
& \left.\cdot\left(1-2^{n-N-1}\right)+e^{-\frac{x_{N, n}^{*} \gamma}{2}} 2^{-2-n}\left(1-2^{1-n}\right)\right] .
\end{aligned}
$$

In this case, the second derivative of $f\left(N ; \boldsymbol{x}_{N}^{*}\right)$ with respect to $x_{N, n}^{*}$ is

$$
\begin{aligned}
\frac{\partial^{2} f\left(N ; \boldsymbol{x}_{N}^{*}\right)}{\partial x_{N, n}^{*}{ }^{2}}= & T^{2} \gamma^{2}\left[e^{-\frac{x_{N, n}^{*} \gamma}{4}} 2^{-1-2 n}\left(1-2^{n-N-1}\right)\right. \\
& \left.+e^{-\frac{x_{N, n^{\gamma}}^{*}}{2}} 2^{-1-n}\left(1-2^{1-n}\right)\right] \\
& >0 .
\end{aligned}
$$

Thus the optimal problem (15) is also convex with respect to $x_{N, n}^{*}$ for the binary orthogonal signals with envelope detection.

The associated set of KKT conditions are

$$
\begin{aligned}
& -T^{2} \gamma e^{-\frac{x_{N, n}^{*} \gamma}{4} 2^{1-2 n}\left(1-2^{n-N-1}\right)-T^{2} \gamma} \\
& \cdot e^{-\frac{x_{N, n}^{*} \gamma}{2}} 2^{-n}\left(1-2^{1-n}\right)+\lambda-\mu_{n}=0
\end{aligned}
$$




$$
\begin{gathered}
\lambda\left(\sum_{n=1}^{N} x_{N, n}^{*}-1\right)=0 \\
\sum_{n=1}^{N} x_{N, n}^{*}=1 \\
\mu_{n} x_{N, n}^{*}=0, \quad n=1, \ldots, N \\
\lambda \geq 0 \quad \mu_{n} \geq 0 \quad x_{N, n}^{*} \geq 0 \quad n=1, \ldots, N .
\end{gathered}
$$

Next we proceed to solve the KKT systems. Multiplying both side of (28) by $x_{N, n}^{*}$ eliminates $\mu_{n}$, and we obtain the following equation

$$
\begin{aligned}
x_{N, n}^{*} & {\left[-T^{2} \gamma e^{-\frac{x_{N, n}^{*} \gamma}{4}} 2^{1-2 n}\left(1-2^{n-N-1}\right)\right.} \\
& \left.-T^{2} \gamma e^{-\frac{x_{N, n}^{*}}{2}} 2^{-n}\left(1-2^{1-n}\right)+\lambda\right]=0 .
\end{aligned}
$$

If $\lambda \geq T^{2} \gamma 2^{-n}\left(1-2^{-N}\right), x_{N, n}^{*}>0$ is impossible since if $x_{N, n}^{*}>0$ then $-T^{2} \gamma 2^{-n}\left(1-2^{-N}\right)+\lambda>0$ and (33) cannot hold. Thus $x_{N, n}^{*}=0$ if $\lambda \geq T^{2} \gamma 2^{-n}\left(1-2^{-N}\right)$.

Now we show that when $\lambda<T^{2} \gamma 2^{-n}\left(1-2^{-N}\right), \mu_{n}$ should be zero. When $\lambda<T^{2} \gamma 2^{-n}\left(1-2^{-N}\right)$, we have $\mu_{n}<0$ if $x_{N, n}^{*}=0$ using (28), which contradicts (32). Thus if $\lambda<$ $T^{2} \gamma 2^{-n}\left(1-2^{-N}\right), x_{N, n}^{*}$ should not be zero. Then to get (31), we have $\mu_{n}=0$ if $\lambda<T^{2} \gamma 2^{-n}\left(1-2^{-N}\right)$. Therefore (28) becomes

$$
\begin{aligned}
& -T^{2} \gamma e^{-\frac{x_{N, n}^{*} \gamma}{4}} 2^{1-2 n}\left(1-2^{n-N-1}\right) \\
& -T^{2} \gamma e^{-\frac{x_{N, n}^{*} \gamma}{2}} 2^{-n}\left(1-2^{1-n}\right)+\lambda=0
\end{aligned}
$$

Then $x_{N, n}^{*}$ is calculated as

$$
x_{N, n}^{*}= \begin{cases}0, & \lambda \geq T^{2} \gamma 2^{-n}\left(1-2^{-N}\right) \\ -\frac{4}{\gamma} \ln (\eta(\lambda, n)), & \lambda<T^{2} \gamma 2^{-n}\left(1-2^{-N}\right) .\end{cases}
$$

where if $n>1, \eta(\lambda, n)$ is defined as

$$
\begin{aligned}
& \eta(\lambda, n):=\left[T \gamma 2^{1-n}\left(1-2^{1-n}\right)\right]^{-1}\left[-T \gamma 2^{1-2 n}\left(1-2^{n-N-1}\right)\right. \\
& \left.+\sqrt{T^{2} \gamma^{2} 2^{2-4 n}\left(1-2^{n-N-1}\right)^{2}+4 \lambda \gamma 2^{-n}\left(1-2^{1-n}\right)}\right]
\end{aligned}
$$

else

$$
\eta(\lambda, 1):=\frac{2 \lambda}{T^{2} \gamma\left(1-2^{-N}\right)} .
$$

More concisely, we have

$$
x_{N, n}^{*}=\max \left\{0,-\frac{4}{\gamma} \ln (\eta(\lambda, n))\right\} .
$$

Define $\phi(\lambda)$ as

$$
\phi(\lambda):=\sum_{n=1}^{N} \max \left\{0,-\frac{4}{\gamma} \ln (\eta(\lambda, n))\right\} .
$$

Finally we show that $\lambda>0$. If $\lambda=0$, then from (28) we have $\mu_{n}<0$ which contradicts (32). Therefore $\lambda=0$, then (29) becomes

$$
\left(\sum_{n=1}^{N} x_{N, n}^{*}-1\right)=0 .
$$

Taking (38) into (40), we have

$$
\phi(\lambda)=1 .
$$

From (39), we know that $\phi(\lambda)$ is a piecewise-linear nonincreasing function of $\lambda$ with a breakpoint at $T^{2} \gamma 2^{-n}\left(1-2^{-N}\right)$, thus the equation has a unique solution. The unique $\lambda$ can be found by the following algorithm.

\section{Algorithm:}

1) Set $i=1$.

2) Calculate

$$
z_{0}=-\frac{4}{\gamma} \sum_{n=1}^{i} \ln \left(\eta\left(T^{2} \gamma 2^{-i}\left(1-2^{-N}\right), n\right)\right),
$$

and

$$
z_{1}=-\frac{4}{\gamma} \sum_{n=1}^{i} \ln \left(\eta\left(T^{2} \gamma 2^{-(i+1)}\left(1-2^{-N}\right), n\right)\right),
$$

If $z_{0} \leq 1$ and $z_{1} \geq 1$, then go to 4 ), else $i=i+1$ and go to 3 ).

3) Search $\lambda$ at $\left[T^{2} \gamma 2^{-(i+1)}\left(1-2^{-N}\right), T^{2} \gamma 2^{-i}\left(1-2^{-N}\right)\right]$ using the bisearch algorithm to satisfy

$$
-\frac{4}{\gamma} \sum_{n=1}^{i} \ln (\eta(\lambda, n))=1 .
$$

4) Calculate $\left\{\boldsymbol{x}_{N_{\text {opt }}}^{*}\right\}$ as follows:

$$
x_{N, n}^{*}= \begin{cases}0, & n>i ; \\ -\frac{4}{\gamma} \ln (\eta(\lambda, n)), & n \leq i .\end{cases}
$$

The algorithm leads to the global optimum since the optimal $\lambda$ is unique. Finally, the optimal solution $\left\{N_{o p t}, \boldsymbol{x}_{N_{\text {opt }}}^{*}\right\}$ can be obtained based on (16). In Section IV, we will find the optimal solution for specific system setups and show the simulation results.

\section{Simulation Results}

This section presents some simulation results to demonstrate the effectiveness of the proposed quantization schemes. In all simulation runs, the observed signal is chosen as Gaussian truncated to the range $[-5,5]$, i.e., $T=5$ with mean 1 . The channel noise is zero-mean Gaussian distributed. All simulation results are averaged from 50,000 independent runs.

Fig. 1 depicts the reconstruction MSE of different quantization schemes with the total receiving SNR $\gamma=20$ at the FC for BPSK signal over AWGN channels. With different quantization bit rate $N$, the optimal energy allocation $\left\{\boldsymbol{x}_{N}^{*}\right\}$ is obtained by solving (26), and the optimal quantization bit rate $N$ is also found to be $N_{o p t}=5$. We plot the simulated 


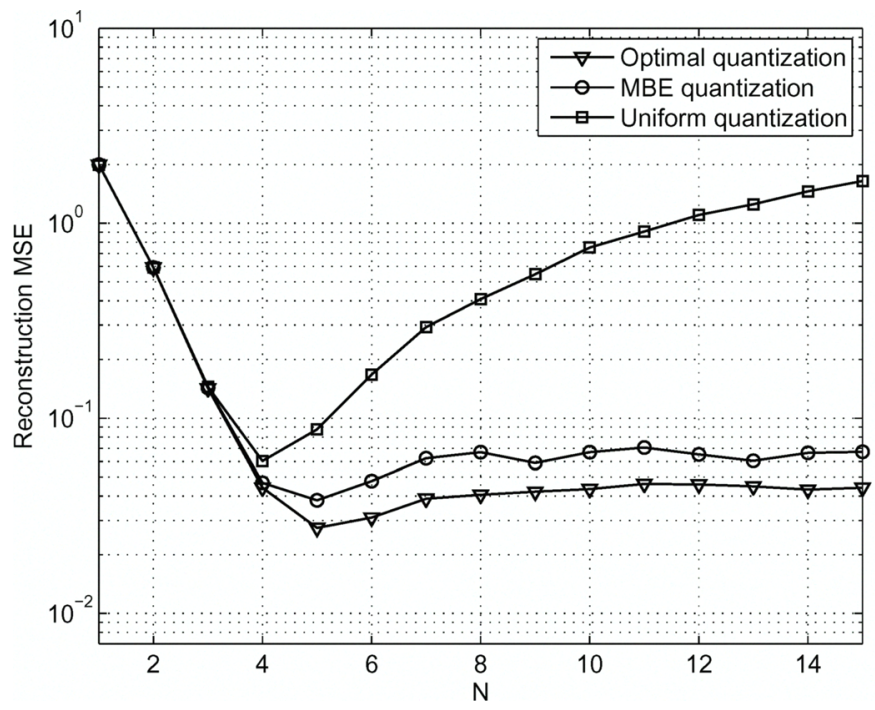

Fig. 1. Reconstruction MSE versus the quantization bit rate per sensor with different energy allocation schemes among bits for BPSK signal.

reconstruction MSEs with the optimal energy allocation $\left\{\boldsymbol{x}_{N}^{*}\right\}$, the MBE energy allocation scheme [10], and the uniform energy allocation scheme, respectively. Fig. 1 shows that the proposed optimal quantization scheme has lower MSE than either the MBE or the uniform energy allocation schemes for BPSK signal over AWGN Channels.

Fig. 2 shows the performance for FSK signal over AWGN channels with the total receiving SNR $\gamma=10$ at the FC. With different quantization bit rate $N$, the optimal energy allocation $\left\{\boldsymbol{x}_{N}^{*}\right\}$ is obtained by solving (35), and the optimal quantization bit rate $N$ is found to be $N_{o p t}=4$. Fig. 2 also demonstrates that the proposed optimal quantization scheme outperforms either the MBE or the uniform energy allocation schemes for FSK signal over AWGN channels.

\section{CONCLUSION}

This paper addressed the optimization of the quantization scheme under strict energy constraint and imperfect transmission to improve the reconstruction MSE performance at the FC. Optimal quantization schemes including the optimal quantization rate as well as the optimal transmission energy allocation, which minimizes the upper bound of the reconstruction MSE, have been proposed for BPSK signal and binary orthogonal signal with envelope detection, respectively. The optimization problems have been proved to be convex and closed-from solutions have been derived in both cases. Simulation results showed that the proposed optimal quantization schemes perform better than either the MBE or the uniform quantization schemes.

\section{REFERENCES}

[1] S. Cui, J.-J. Xiao, A. J. Goldsmith, Z.-Q. Luo, and H. V. Poor, "Estimation diversity and energy efficiency in distributed sensing", IEEE Trans. Signal Process., vol. 55, no. 9, pp. 4683-4695, Sep. 2007.

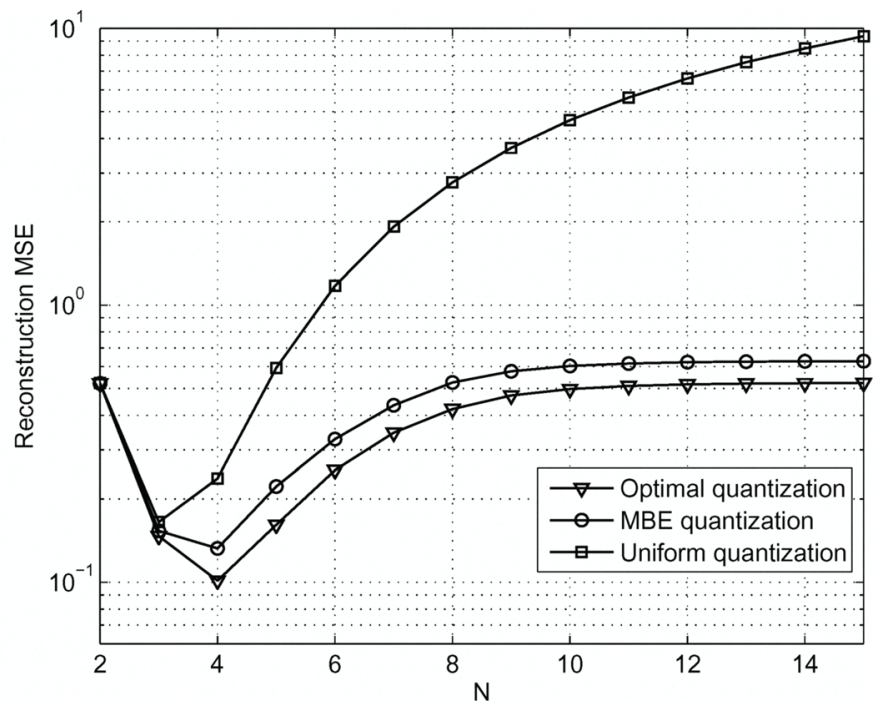

Fig. 2. Reconstruction MSE versus the quantization bit rate per sensor with different energy allocation schemes among bits for FSK signal.

[2] J. -J. Xiao, A. Ribeiro, Z. -Q. Luo and G. B. Giannakis, "Distributed compression-estimation using wireless sensor networks," IEEE Trans. Signal Process., vol. 23, no. 4, pp. 27-41, Jul. 2006.

[3] Z. -Q. Luo, "Universal decentralized estimation in a bandwidth constrained sensor network," IEEE Trans. Inf. Theory, vol. 51, no. 6, pp. 2210-2219, Jun. 2005.

[4] A. Ribeiro and G. B. Giannakis, "Bandwidth-constrained distributed estimation for wireless sensor networks-part II: Unknown probability density function,” IEEE Trans. Signal Process., vol. 54, no. 7, pp. 11311143, Jul. 2006.

[5] Y. Huang and Y. Hua, "Multihop progressive decentralized estimation in wireless sensor networks," IEEE Signal Process. Lett., vol. 14, no. 12, pp. 1004-1007, Dec. 2007.

[6] T. C. Aysal and K. E. Barner, "Constrained decentralized estimation over noisy channels for sensor networks," IEEE Trans. Signal Process., vol. 56, no. 4, pp. 1398-1410, Apr. 2008.

[7] J. -J. Xiao, S. Cui, Z. -Q. Luo and A. J. Goldsmith, "Power scheduling of universal decentralized estimation in sensor networks," IEEE Trans. Signal Process., vol. 54, no. 2, pp. 413-422, Feb. 2006.

[8] J.-Y. Wu, Q.-Z. Huang and T.-S. Lee, "Minimal energy decentralized estimation via exploiting the statistical knowledge of sensor noise variance," IEEE Trans. Signal Process., vol. 56, no. 5, pp. 2171-2176, May 2008.

[9] H. Senol, and C. Tepedelenlioglu, "Distributed estimation over parallel fading channels with channel estimation error," Proc. IEEE Int. Conf. Acoust., Speech Signal Process. (ICASSP) 2008, Las Vegas, Mar. 31 2008 - Apr. 4 2008, pp. 3305-3308.

[10] X. Luo and G. B. Giannakis, "Energy-constrained optimal quantization for wireless sensor networks," Proc. 1st IEEE Annual Communications Society Conference on Sensor and Ad Hoc Communications and Networks (SECON '04), pp. 272-278, Santa Clara, Calif, USA, Oct. 2004.

[11] J. G. Proakis, Digital Communications, McGraw-Hill, 4th Edition, 2001.

[12] S. Boyd and L. Vandenberghe, Convex Optimization, Cambridge, U.K.: Cambridge Unv. Press, 2003. 